Open Access Full Text Article

ORIGINAL RESEARCH

\title{
Increased Oxidative Stress, Loop Gain And The Arousal Threshold Are Clinical Predictors Of Increased Apnea Severity Following Exposure To Intermittent Hypoxia
}

This article was published in the following Dove Press journal:

Nature and Science of Sleep

\author{
Gino S Panzal,2,* \\ Raichel M Alex ${ }^{1,2, *}$ \\ Sanar S Yokhana (D) ${ }^{1,2}$ \\ Dorothy S Lee Pioszak ${ }^{1,2}$ \\ M Safwan Badr ${ }^{1-3}$ \\ Jason H Mateikal-3 \\ 'Research and Development, John D. \\ Dingell Veterans Affairs Medical Center, \\ Detroit, MI 4820I, USA; '² Department of \\ Physiology, Wayne State University \\ School of Medicine, Detroit, MI 4820I, \\ USA; ${ }^{3}$ Department of Internal Medicine, \\ Wayne State University School of \\ Medicine, Detroit, MI 4820I, USA \\ *These authors contributed equally to \\ this work
}

Purpose: We determined if oxidative stress prior to sleep onset is correlated to loop gain (LG) and the arousal threshold (AT) during non-rapid eye movement (NREM) sleep. We also explored if LG and AT are correlated with apnea severity and indices of upper airway collapsibility during NREM sleep.

Methods: Thirteen male participants with obstructive sleep apnea (apnea-hypopnea index $>5$ events/hr) were administered an antioxidant or placebo cocktail while exposed to mild intermittent hypoxia in the awake state. Thereafter, loop gain and measures of arousal, apnea severity and upper airway collapsibility were ascertained during NREM sleep.

Results: Modification in oxidative stress (i.e., 8-hydroxy-2-deoxyguanosine) prior to sleep onset was correlated to LG $(\mathrm{r}=0.8, \mathrm{P}=0.003)$, the number $(\mathrm{r}=0.71, \mathrm{P}=0.01)$ and duration $(r=0.63, P=0.04)$ of apneic events and the percentage of time breathing was stable $(r=-0.66$, $\mathrm{P}=0.03$ ) during sleep. Using a forward stepwise regression analysis, our results showed that $\mathrm{LG}$, AT, the ventilatory response to arousal and nadir end-tidal carbon dioxide were determinants of the apnea-hypopnea index ( $\mathrm{P}$ value range $=0.04-0.001$ ). In addition, the AT was a predictor of measures of upper airway collapsibility, including the hypopnea/apnea + hypopnea ratio and the degree of flow reduction that accompanied hypopneic events $(\mathrm{P}<0.001)$.

Conclusion: Modifications in oxidative stress following exposure to intermittent hypoxia during wakefulness are positively associated with loop gain and apnea severity during NREM sleep. Moreover, an increase in the arousal threshold is a predictor of increased upper airway collapsibility.

Keywords: mild intermittent hypoxia, non-rapid eye movement sleep, arousal, oxidative stress, loop gain

\section{Introduction}

Exposure to brief episodes of mild intermittent hypoxia leads to a progressive increase in the chemoreflex response to hypoxia (i.e., progressive augmentation of the hypoxic ventilatory response) $)^{1-4}$ and long-term facilitation of ventilation ${ }^{1,5-8}$ and upper airway muscle activity ${ }^{5,9,10}$ in humans during wakefulness when carbon dioxide is maintained above baseline levels. Modifications in the chemoreflex response are reflected in measures of controller gain, which is one of the two primary components of loop gain (see section "Loop Gain And Arousal Threshold Analysis" for further explanation). Modifications in controller gain/loop gain during
Correspondence: Jason H Mateika John D. Dingell VA Medical Center, 4646 John R (I IR), Room 4333, Detroit, MI 4820I, USA

Tel + I $3|3576448|$

$\mathrm{Fax}+1313576$ III2

Email jmateika@med.wayne.edu 
wakefulness, following exposure to mild intermittent hypoxia, may extend into sleep and impact apnea severity. Indeed, loop gain and the arousal threshold are increased during non-rapid eye movement sleep immediately following exposure to mild intermittent hypoxia during wakefulness. $^{11}$

Loop gain measures during sleep following exposure to mild intermittent hypoxia during wakefulness may be influenced by free radicals which enhance the carotid body sensory response to hypoxia ${ }^{11}$ and initiate carotid sensory nerve long-term facilitation. ${ }^{12}$ Indeed, treatment with potent scavengers of superoxide anions abolishes the amplified carotid sensory response to hypoxia and carotid sensory nerve long-term facilitation, ${ }^{11-13}$ as well as, longterm facilitation of both phrenic and hypoglossal nerve activity $^{14}$ in rats. In addition, chemoreflex sensitivity to hypoxia and hypercapnia along with the amplitude of ventilatory long-term facilitation is reduced in humans with obstructive sleep apnea following administration of an antioxidant cocktail. ${ }^{6}$ Given these findings, we hypothesized that measures of oxidative stress following exposure to mild intermittent hypoxia in the awake state are positively correlated to measures of loop gain and the arousal threshold during non-rapid eye movement sleep. To test this hypothesis, an antioxidant or placebo cocktail was administered to participants with obstructive sleep apnea during exposure to acute intermittent hypoxia immediately prior to sleep.

Increases in loop gain during sleep may be coupled to an increased ventilatory drive to chemical stimuli (i.e., hypoxia and hypercapnia) and arousal at the termination of an apneic event, inducing hypocapnia and a subsequent apnea upon the resumption of sleep. ${ }^{15}$ An increase in apnea duration might also occur concurrently with increases in loop gain, because carbon dioxide levels may be driven further below the apneic threshold leading to an increase in the arousal threshold. ${ }^{15,16}$ These suggestions are supported by recent findings which showed that loop gain and the arousal threshold were positively correlated to increases in apnea frequency and duration. ${ }^{15}$

Based on these findings, we were interested in examining further the relationship between loop gain or the arousal threshold, determined using a model-based approach, and other clinical markers of sleep apnea severity measured during non-rapid eye movement sleep, following exposure to mild intermittent hypoxia during wakefulness. We hypothesized that the arousal threshold and loop gain would be correlated to the ventilatory response to arousal and the accompanying nadir of end-tidal carbon dioxide. We also hypothesized that the increased loop gain and arousal threshold would be correlated to indices of airway collapsibility (i.e., the degree of airflow limitation and the percentage of apnea or hypopnea events as a percentage of total events).

\section{Methods}

\section{Protocol Overview}

The Institutional Review Boards of Wayne State University and John D. Dingell Veterans Affairs Medical Center approved the protocol. The protocol conformed to the standards set by the Declaration of Helsinki. Untreated male participants $(n=13)$ with obstructive sleep apnea, that were healthy otherwise (i.e., not on any other medication, absence of heart and lung disease, hypertension, morbid obesity), completed the study after obtaining written informed consent. The participant's visited the laboratory 4 times. Prior to each visit, participants were instructed to pass up caffeinated beverages and food at least $4 \mathrm{hrs}$ before the study and to have at least $7 \mathrm{hrs}$ of sleep. Participants arrived at the laboratory at approximately 6:00 PM on visits 3 and 4 .

During visit 1, a physical exam, 12-lead electrocardiogram and blood pressure measurements were completed. During this visit, participants were also exposed to two 4min episodes of hypoxia so that they would be familiar with the protocol and equipment. During the second visit, participants completed a baseline sleep study to substantiate the occurrence of obstructive sleep apnea. On the third and fourth visits that were separated by one week, intermittent hypoxia was administered, while the participants were in the supine position (see section "Intermittent Hypoxia Protocol" for further details). Prior to the administration of intermittent hypoxia, venous blood $(8 \mathrm{~mL})$ was sampled. Subsequently, the oral components of the antioxidant (see section "Antioxidant Cocktail" below for details concerning the antioxidant cocktail) or placebo cocktail were administered. Sixty to ninety minutes after administering the oral component of the antioxidant or placebo cocktail, an intravenous component was administered before exposure to intermittent hypoxia. The intravenous component of the antioxidant or placebo cocktail was also administered midway through the intermittent hypoxia protocol. Eight milliliters of blood were also drawn following exposure to intermittent hypoxia. The antioxidant and placebo cocktail were dispensed using a randomized approach. Once mild 
intermittent hypoxia was administered to the participants on Visits 3 and 4, they completed a sleep study.

\section{Polysomnography Studies}

Participants were in the supine position throughout the sleep studies completed on visits 2,3 and 4. During the sleep studies an electroencephalogram (C3/A2, C4/A1, O1/A2, O2/A1), submental and tibialis anterior electromyogram, three-lead electrocardiogram and electrooculograms were measured. Inductive plethysmography (Respitrace, Ambulatory Monitoring, Inc., Ardsley, NY, USA) was used to measure chest wall and abdominal movements. A pneumotachometer (Model RSS100-HR, Hans Rudolph Inc., Kansas, MO, USA) connected to a nasal mask was employed to measure flow and inspiratory and expiratory time. Esophageal pressure was monitored using a transducer-tipped catheter (MPC-500, Millar, Inc., Houston, TX, USA) to corroborate the detection of breathing events. A pulse oximeter (Biox 3700, Ohmeda Corp., Laurel, MD, USA) was used to monitor oxygen saturation.

A software package (Gamma v. 4.0, Astro-Med Inc., West Warwick, RI, USA) was used to capture the physiological variables that were analog to digitally converted and sampled at $100 \mathrm{~Hz}$ per channel.

\section{Intermittent Hypoxia Protocol}

Participants breathed room air for 10 mins to establish baseline values of minute ventilation and the partial pressure of end-tidal carbon dioxide. Thereafter, carbon dioxide was elevated $4 \mathrm{mmHg}$ above baseline and this new baseline was maintained for 15 mins. Subsequently, the increased level of carbon dioxide was maintained for the remainder of the protocol. The increase in carbon dioxide was initiated because ventilatory long-term facilitation is apparent in humans when carbon dioxide is maintained above baseline but is not apparent when values are less than baseline. ${ }^{3,5}$ After establishing the new baseline, participants were exposed to twelve 4-min episodes of hypoxia with 4 mins of normoxia interposed amid the episodes. Throughout the hypoxic episodes, participants inspired a mixture of gas consisting of $8 \%$ oxygen and balance nitrogen. Additional oxygen and carbon dioxide were added to the gas mixture that was inspired to sustain the partial pressure of end-tidal oxygen at $50 \mathrm{mmHg}$ and to maintain carbon dioxide above resting values. At the end of each episode, a single breath of $100 \%$ oxygen was inspired to re-establish the partial pressure of end-tidal oxygen in the normoxic range. After the last hypoxic episode, ventilation was monitored for 30 mins while participants breathed room air. During the 30-min recovery period, carbon dioxide levels were maintained $4 \mathrm{mmHg}$ above resting baseline levels.

During the protocol, participants wore a face mask that was attached to a pneumotachograph (model RSS100-HR, Hans Rudolph, Inc., Shawnee, KS, USA), which monitored changes in ventilation breath-by-breath. The pneumotachograph was connected to a two-way valve. The inspiratory end of the valve was affixed to a five-way stopcock. Participant's inspired room air or the contents from one of the two bags connected to the stopcock. One bag contained $8 \%$ oxygen-balance nitrogen to decrease oxygen to $50 \mathrm{mmHg}$ at the beginning of each hypoxic episode, and the second bag was comprised of $100 \%$ oxygen to quickly introduce normoxia at the end of each hypoxic episode. Furthermore, the output from a flowmeter was connected to a stopcock port that was attached to the two-way valve. Cylinders containing $100 \%$ oxygen and $100 \%$ carbon dioxide were connected to the flowmeter. Consequently, $100 \%$ oxygen and $100 \%$ carbon dioxide could be added to the $8 \%$ oxygen-balance nitrogen to maintain desired oxygen and carbon dioxide levels. Oxygen and carbon dioxide analyzers (model 17518 and model 17515, Vacumetrics, Inc., Ventura, CA, USA) were used to sample end-tidal oxygen and carbon dioxide from face mask ports. A pulse oximeter (Biox 3700, Ohmeda Corp., Laurel, MD, USA) was used to monitor oxygen saturation and a three-lead electrocardiogram was monitored. The physiological signals were analog to digitally converted using a 16-bit analog-to-digital converter (ATMIO-16XE-50, National Instruments, Austin, TX, USA) and data analysis was completed using software designed for this intention. Breath-by-breath minute ventilation, $\mathrm{P}_{\mathrm{ET}} \mathrm{CO}_{2}$ and $\mathrm{P}_{\mathrm{ET}} \mathrm{O}_{2}$ were calculated using the software.

\section{Antioxidant Cocktail}

The pharmacist at John D. Dingell VA Medical Center formulated and randomized the antioxidant and placebo cocktail. Laboratory employees were blinded to the randomization process. The antioxidant cocktail consisted of $60 \mathrm{mg}$ of coenzyme Q10 (Cell Tech International Inc., Klamath Falls, OR, USA), $400 \mathrm{mg}$ of superoxide dismutase (P. L. Thomas and Co. Inc., Morristown, NJ, USA), 200 IU of vitamin E (Goldline Laboratories Inc., Miami, FL, USA) and vitamin C (2 doses of $1 \mathrm{~g}$ in $50 \mathrm{~mL}$ of $0.9 \mathrm{NaCl}$ ) (Hospira Inc., Rocky Mount, NC, USA). The rationale for selecting each component of the cocktail has been outlined in work previously published 
from our laboratory. ${ }^{6}$ As mentioned in the protocol overview, an intravenous dose of vitamin $\mathrm{C}$ was administered to the participants immediately before exposure to intermittent hypoxia. An intravenous infusion of $0.9 \mathrm{NaCl}$ at $10 \mathrm{~mL} / \mathrm{hr}$ was initiated in the antecubital fossa before administration of the vitamin $\mathrm{C}$. Thereafter, vitamin $\mathrm{C}$ was infused over a 20min interval. Vitamin $\mathrm{C}$ was infused immediately prior to intermittent hypoxia exposure because peak plasma levels normally occur 90 mins following infusion with an approximately 30-min half-life. The oral component of the sham cocktail was comprised of cellulose capsules and the intravenous component of a $0.9 \% \mathrm{NaCl}$ infusion. The oral component of the placebo cocktail was consumed with $170 \mathrm{~g}$ of yogurt. The time frame of administration of the antioxidant and placebo cocktail was identical.

\section{Data Analysis}

\section{Measures Of Oxidative Stress}

Glutathione (GSH) was used as a biomarker of antioxidant capacity. Glutathione prevents damage to cellular elements caused by peroxides, free radicals, heavy metals and lipid peroxides. 8-hydroxy-2-deoxy guanosine (8-OHdG) and 8isoprostane, which are well-established biomarkers of oxidative stress, were also measured. Previous studies have revealed that the measured biomarkers are modified in participants with OSA. ${ }^{17-21}$ Oxidative stress was ascertained from plasma samples acquired from EDTA treated venous blood centrifuged at $1000 \mathrm{~g}$ for $10 \mathrm{mins}$ at $4^{\circ} \mathrm{C}$. After this process was completed, plasma was stored at $-80^{\circ} \mathrm{C}$ pending the measurement of oxidative stress using standard assays (Cayman Chemical Co., Ann Arbor, MI, USA). Only full data sets (i.e., baseline and recovery measures of a given biomarker for both the antioxidant and placebo trials) were used for the analyses. Consequently, different $n$ values were associated with each biomarker. GSH and 8-OHdG measures were obtained from all but one participant $(n=12)$ and complete measures of 8-isoprostane were obtained from 11 participants. Based on the $n$ values, we considered G8-OHdG to be the primary measure of oxidative stress and provide detailed results on this biomarker. In addition, this variable was used in the correlation analysis (see sections "Statistical Analysis" and "Results").

\section{Polysomnography}

Published criteria ${ }^{22}$ were used to stage sleep and score respiratory events and arousals. A $\geq 90 \%$ reduction in airflow for $\geq 10$ s defined apnea events, while a hypopnea was defined by $a \geq 30 \%$ reduction in airflow. Events were classified as respiratory-related if the reduction in airflow was accompanied by either an arousal or a $\geq 3 \%$ drop in oxygen saturation. Gradual increases in esophageal pressure, and thoracic and abdominal movements were used to identify obstructive events. Arousals were scored if electroencephalography frequency and amplitude changed significantly for 3 or more seconds. The beginning and end of arousals were identified to confirm that this standard was met. In addition to measures obtained using a customized MATLAB program (see section "Loop Gain And Arousal Threshold Analysis"), the ventilatory response to arousal (i.e., the greatest tidal volume measured from the initial 3 breaths recorded after an event), apnea severity and degree of airway collapsibility were determined from the sleep studies completed during the antioxidant and placebo trials. These measures included the end-tidal level of carbon dioxide associated with the breath of greatest magnitude following arousal from breathing events, the apnea/ hypopnea index, apneic event duration, the average decrease in oxygen saturation during events, the arousal index, the hypopnea/apnea + hypopnea ratio and the degree of flow limitation during hypopneic events (i.e., the percentage decrease in flow compared to measures of stable baseline breathing).

\section{Loop Gain And Arousal Threshold Analysis}

A MATLAB (Mathworks, Natick, MAS, USA) program was used to quantify loop gain [see next paragraph for list of components and Figure 1 in reference ${ }^{23}$ to obtain a pictorial description of the components] and the arousal threshold. $^{23-25}$ Seven-minute windows of non-rapid eye movement or rapid eye movement sleep were distinguished. The sleep stage that comprised greater than $50 \%$ of the window was identified. Windows that were not characterized by a dominant sleep stage were eliminated. The window duration (i.e., 7 mins) was selected to allow approximately 10 obstructive events to transpire. The number of possible events was based on an average inter-event interval of approximately $40 \mathrm{~s}$. This interval was judged to be appropriate to distinguish the chemical and arousal contribution to the ventilatory drive output during and after an event.

A breath by breath time sequence was created for each window and breaths associated with an EEG arousal and/ or flow limitation were identified. Thereafter, the program measured loop gain and the arousal threshold. The program $^{23}$ was created on the supposition that ventilatory control is disrupted during breathing events leading to 


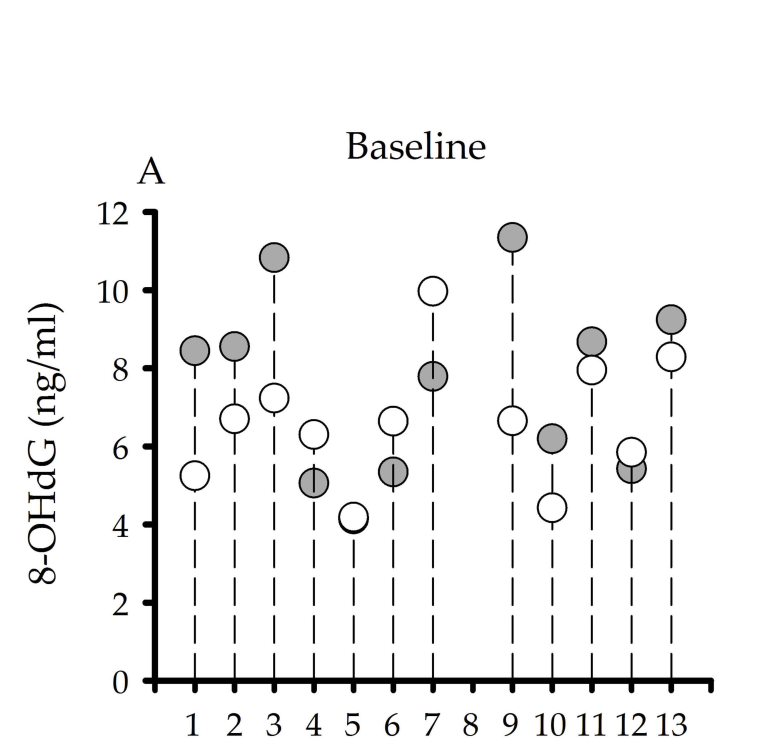

Participant

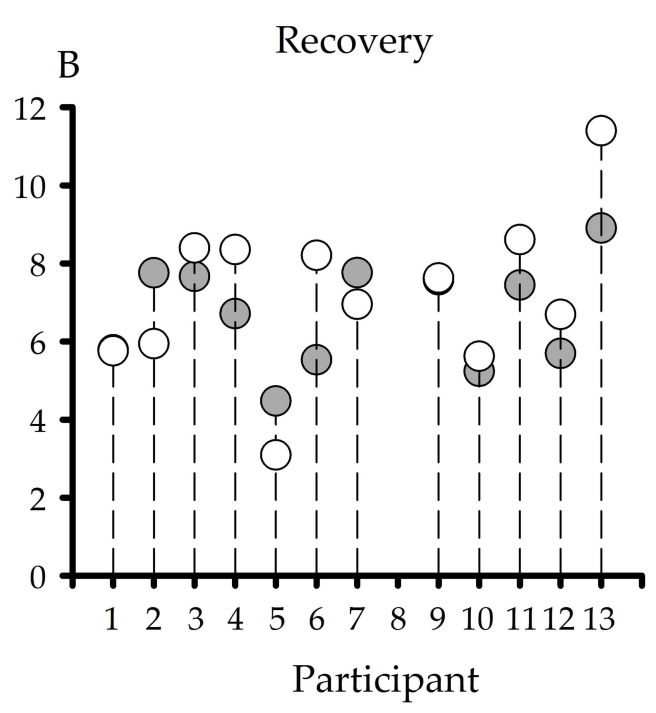

Participant

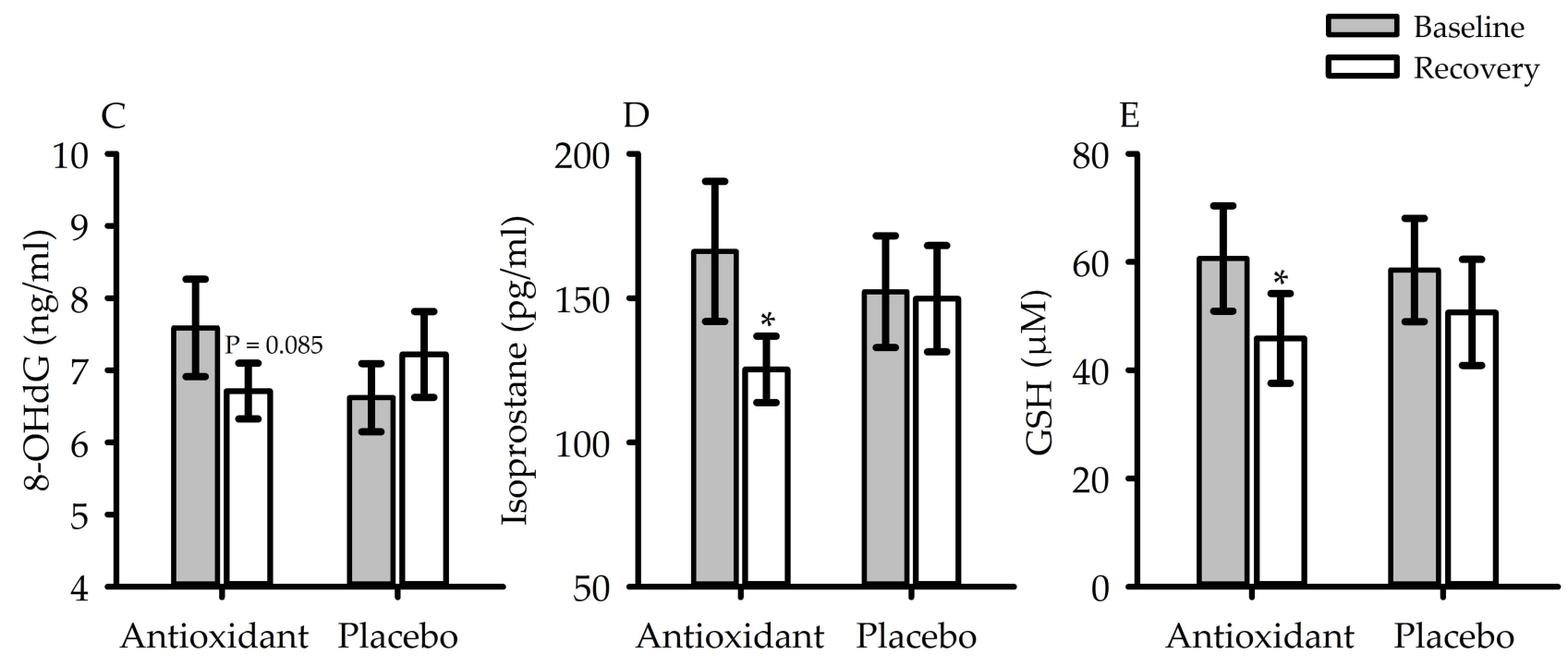

Figure I Scatterplots showing individual measures of 8-hydroxy-2-deoxy guanosine (8-OHdG) $(n=12)$ during $(\mathbf{A})$ baseline and $(\mathbf{B})$ recovery of the antioxidant (gray circles) and placebo (white circles) trials. Bar graphs which show average measures of $(\mathbf{C}) 8-O H d G(n=12),(D)$ 8-isoprostane $(n=I I)$ and $(E)$ glutathione $(G S H)(n=I 2)$. Measures of 8-OHdG, 8-isoprostane and GSH decreased during recovery compared to baseline during the antioxidant trial. In contrast, these same measures following exposure to intermittent hypoxia during the placebo trial were either increased or similar to baseline measures. Nonetheless, measures of oxidative stress during recovery of the antioxidant and placebo trial were similar (for explanation, see section "Oxidative Stress, Loop Gain, Arousal Threshold And Measures Of Apnea Severity"). *indicates as significantly less than baseline $P<0.05$; Paired $t$-tests were used to make comparisons between baseline and recovery of the antioxidant or placebo trials.

increases in carbon dioxide and decreases in oxygen that lead to an augmented ventilatory drive. The increased drive is revealed in the degree of hyperventilation that occurs after opening of the airway that follows termination of an obstructive event. Accordingly, ventilatory drive is modeled as the sum of the ventilatory response to chemical stimuli (i.e., increases in carbon dioxide and decreases in oxygen that accompany apnea and hypopneas) and arousal. ${ }^{23}$ The time sequence of the ventilatory response to chemical stimuli is modeled using limits that indicate the lung to chemoreceptor circulation time (i.e., time delay), the time interval associated with buffering of carbon dioxide in the lung and tissues (i.e., time constant) and the gain of the response. ${ }^{23}$ These limits coupled with the ventilatory response to arousal were adjusted until ventilatory drive fits the ventilation that was quantified when the airway was unobstructed. These limits were then employed to compute loop gain at the natural frequency $\left(\mathrm{LG}_{\mathrm{n}}\right)$ of obstructive events. Loop gain was also calculated at a frequency of 1 cycle per minute $\left(L_{1}\right)$ (i.e., 60 events/ 
hour) for each window. This frequency was selected to be consistent with the timing of apneic events and because this frequency has been typically reported in other published findings. For each window, the ventilatory drive measured immediately before an electroencephalogram arousal was identified. The mean of the ventilatory drive values was considered to be the arousal threshold. The time delay, time constant, loop gain, arousal threshold and the ventilatory response to arousal were averaged for each participant.

Average values for the physiological variables (see sections "Polysomnography" and "Loop Gain And Arousal Threshold Analysis") were ascertained for the initial $3 \mathrm{hrs}$ of sleep. This time frame was selected because the effects of the antioxidant cocktail on loop gain and the arousal threshold would likely be most evident during this portion of the sleep period. Analysis of the physiological variables was not established for rapid eye movement sleep because this stage was evident in only a few of the participants and was not reliably apparent during both the antioxidant and placebo trials. To compare the measures obtained during non-rapid eye movement sleep, we controlled for sleep stage within a given participant across trials (i.e., antioxidant vs placebo), since sleep stage has an impact on arousal threshold, ${ }^{26}$ loop gain ${ }^{25}$ and measures of apnea severity. ${ }^{27}$ In six of the participants, measures of loop gain, arousal threshold and measures of apnea severity were compared in N1 of non-rapid eye movement sleep, because a similar percentage of the total time was composed of this arousal state during the antioxidant and placebo trial. The same measures were compared in N2 in seven participants. In addition to comparing absolute values between trials, data collected during the antioxidant trial, including measures of oxidative stress, were expressed as a fraction of the values collected during the placebo trials. The rationale for expressing the antioxidant data as a fraction of the placebo data are outlined in detail in the results section (see section "Oxidative Stress, Loop Gain, Arousal Threshold And Measures of Apnea Severity"). We also completed univariate and stepwise correlation analysis (see section "Statistical Analysis" for details). This analysis was completed without standardizing for sleep stage since comparisons were made for a given individual and trial.

\section{Statistical Analysis}

A student's paired $t$-test was used to compare absolute measures of loop gain, arousal threshold and ventilatory response to arousal during the initial $3 \mathrm{hrs}$ of non-rapid eye movement sleep of the antioxidant and placebo trials. A paired $t$-test was also used to compare measures of oxidative stress during baseline and recovery of the antioxidant or placebo trial. The same test was used to compare measures of oxidative stress following exposure to intermittent hypoxia in the antioxidant and placebo trials. Pearson's correlation coefficient was used to correlate one measure of oxidative stress, 8-OHdG (see section "Blood Chemistry" for rationale for selecting this variable), with measures of loop again, arousal threshold, the ventilatory response to arousal, measures of apnea severity and airway collapsibility. Data used in this correlation were measures obtained during the antioxidant trial that were expressed as a fraction of the measures obtained during the placebo trials.

Pearson's correlation coefficient was also used to correlate either loop gain or the arousal threshold with measures related to the arousal response, apnea severity or measures of airway collapsibility. Correlations using loop gain at 1 cycle/min are presented since this variable has been typically presented in prior publications. ${ }^{23-25}$ Comparable correlations were also evident using measures of natural loop gain. Thereafter, forward stepwise regression analyses were used to identify the variables that predicted indices of apnea severity. The arousal threshold, ventilatory response to arousal, the partial pressure of end-tidal carbon dioxide following arousal, $\mathrm{LG}_{1}, \mathrm{LG}_{\mathrm{n}}$, age and body mass index were used to predict indices of apnea severity (i.e., apnea/ hypopnea index, the decrease in oxygen saturation during an event) and airway collapsibility (i.e., hypopnea/apneahypopnea ratio and average flow limitation during hypopneic events). In all cases, body mass index was forced into the equation.

\section{Results}

Anthropometric measures for the 13 participants that completed the study are shown in Table 1. In addition, the apnea/hypopnea index for the initial $3 \mathrm{hrs}$ of non-rapid eye movement sleep recorded during the baseline, antioxidant and placebo trials are included in Table 1.

\section{Blood Chemistry}

Figure 1 shows individual (Figure 1A and $\mathrm{B}$ ) and average (Figure 1C) measures of 8-OHdG before (i.e., baseline Figure 1A) and after (i.e., recovery - Figure 1B) exposure to intermittent hypoxia during the antioxidant and placebo trials $(n=12)$. During the antioxidant trial, the reduction in oxidative stress during the recovery period compared to baseline approached statistical significance in the case of 
Table I Anthropometric Measures

\begin{tabular}{|l|l|}
\hline Variable & \\
\hline Number of Participants & 13 \\
Age $(\mathrm{yr})$ & $28.2 \pm 1.7$ \\
Height $(\mathrm{cm})$ & $181.6 \pm 0.99$ \\
Weight $(\mathrm{kg})$ & $92.5 \pm 3.4$ \\
Body Mass Index $\left(\mathrm{kg} / \mathrm{m}^{2}\right)$ & $27.8 \pm 0.6$ \\
Systolic Blood Pressure $(\mathrm{mmHg})$ & $1 \mathrm{I} 8.9 \pm 2.4$ \\
Diastolic Blood Pressure $(\mathrm{mmHg})$ & $80.5 \pm 3.1$ \\
Epworth Sleepiness Scale & $10.8 \pm 1.4$ \\
Stanford Sleepiness Scale & $2.9 \pm 0.4$ \\
Race & $4 \mathrm{AA}, 9 \mathrm{C}$ \\
\hline Apnea/Hypopnea Index (events/hr) & \\
\hline Baseline & $36.5 \pm 6.1$ \\
Antioxidant & $43.3 \pm 6.8$ \\
Placebo & $48.5 \pm 6.4$ \\
\hline
\end{tabular}

Notes: Values are means \pm SE. The apnea/hypopnea index reported is for the initial three hours of sleep.

Abbreviations: AA, African American; C, Caucasian

8-OHdG $(\mathrm{P}=0.085)$ (Figure $1 \mathrm{C})$ and was statistically significant in the case of 8-isoprostane $(\mathrm{P}=0.04)$ (Figure 1D) and GSH $(\mathrm{P}=0.01)$ (Figure 1E). Despite this reduction, the individual data show that oxidative stress during recovery (Figure 1B) of the antioxidant trial was reduced in 7 of 12 participants compared to the placebo. On the other hand, oxidative stress during the recovery period of the antioxidant trial was similar or higher compared to placebo in the remaining 5 participants. In 3 of 5 participants, oxidative stress decreased from baseline to recovery during the antioxidant trial (see participants 1,2 and 9). However, because oxidative stress was greater during baseline of the antioxidant trial, compared to placebo (Figure 1A), oxidative stress remained greater or was similar during recovery compared to the placebo (Figure 1B). Accordingly, oxidative stress following exposure to mild intermittent hypoxia was not statistically different during the antioxidant and placebo trials.

\section{Oxidative Stress, Loop Gain, Arousal Threshold And Measures Of Apnea Severity}

Based on blood chemistry, it was not surprising that loop gain $\left(\mathrm{LG}_{\mathrm{n}}: \mathrm{P}=0.40, \mathrm{LG}_{1}: \mathrm{P}=0.28\right)$, arousal threshold $(\mathrm{P}=0.97)$ and the ventilatory response to arousal $(\mathrm{P}=0.59)$ during nonrapid eye movement sleep was similar across the antioxidant and placebo trials. However, because oxidative stress following exposure to intermittent hypoxia did differ within participants, in some cases being lower $(\mathrm{n}=7)$ and in other cases being higher during the recovery period of the antioxidant trial (Figure 1), we determined if changes in oxidative stress between the antioxidant and placebo trial (i.e., expressed as a fraction of placebo) were correlated with changes in loop gain, arousal threshold and other measures of apnea severity and airway collapsibility. Figure 2 shows that changes in $8-\mathrm{OHdG}$ were positively correlated to the apnea-hypopnea index, the number of events detected within each 7-min segment of non-rapid eye movement sleep and apnea duration. In contrast, changes in $8-\mathrm{OHdG}$ were negatively correlated to the percentage of time breathing was stable within each 7-min segment, when data from one participant were removed because it was a clear outlier (Figure 2 black circle). If the outlier was included the correlation was not significant $(r=0.54, p=0.07)$. In addition, 8-OHdG was positively correlated to the number of arousals detected within each 7-min segment and the natural loop gain. The correlation between 8 -OHdG and the natural loop gain remained significant $(0.77, \mathrm{p}=0.004)$ when the outlier (black circle) shown in Figure 2 was included in the analysis.

\section{Loop Gain And Arousal Threshold As Clinical Markers Of Apnea Severity}

Given that variations in oxidative stress were correlated to loop gain and the arousal threshold, we explored whether these latter measures were correlated to measures of the arousal response, apnea severity or airway collapsibility for each participant. The univariate correlation analysis showed that the arousal threshold and $\mathrm{LG}_{1}$ were positively correlated to the respiratory-related arousal index (Figures 3 and 4). This was also the case for the average number of arousals that were detected from the 7-min segments (AT: $r=0.49, p=0.001 \&$ $\left.\mathrm{LG}_{1}: \mathrm{r}=0.64, \mathrm{p}=0.0004\right)$ used to measure the arousal threshold and loop gain. The arousal threshold and $\mathrm{LG}_{1}$ were positively correlated to the ventilatory response to an arousal and negatively correlated to the partial pressure of end-tidal carbon dioxide following an arousal (Figures 3 and 4). Likewise, the arousal threshold and $\mathrm{LG}_{1}$ were correlated to indices of apnea severity. More specifically, the arousal threshold and $\mathrm{LG}_{1}$ were positively correlated to the apnea/hypopnea index for the $3-\mathrm{hr}$ sleep period. This was also the case for the number of events detected within each 7-min segment of non-rapid eye movement sleep (AT: $r=0.71, p=0.0001 \& L_{1}: r=0.65$, $\mathrm{p}=0.0004$ ) used to measure the arousal threshold and loop gain. Similarly, the arousal threshold was positively correlated to event duration (Figure 3) and the arousal threshold and $\mathrm{LG}_{1}$ 
Apnea/Hypopnea Index

(fraction of placebo)

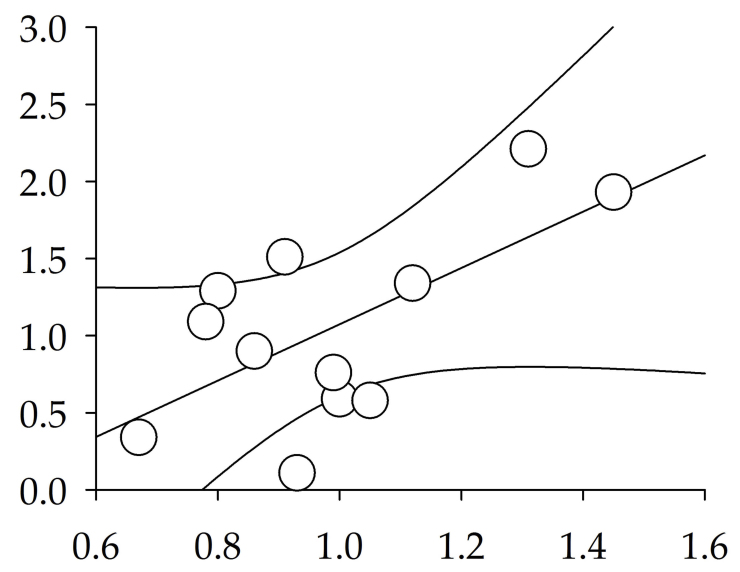

Event Duration

(fraction of placebo)

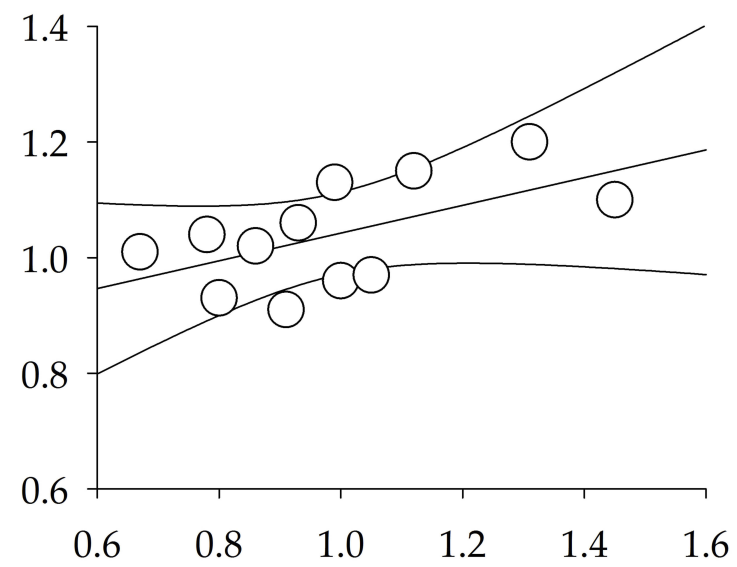

\# of events in 7 minute

$\mathrm{r}=0.71$

segment (fraction of placebo)
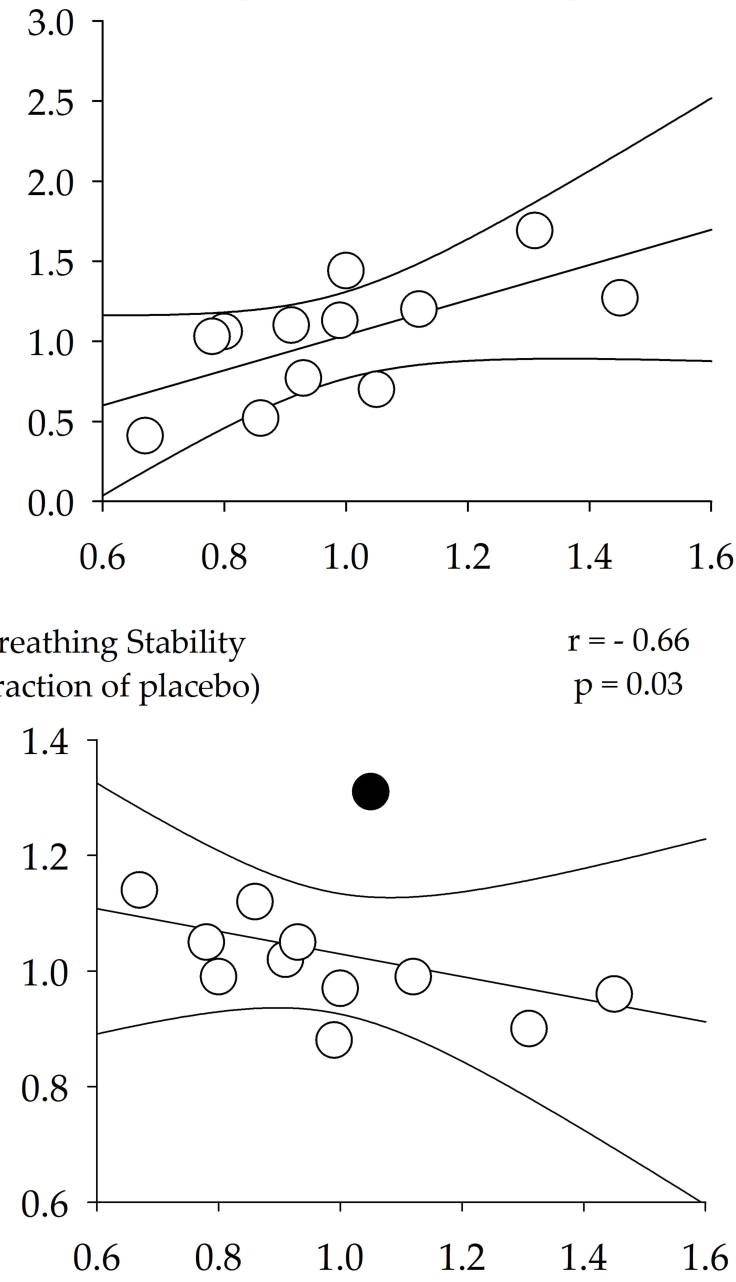

\# of arousals in 7 minute segment

(fraction of placebo)

$\mathrm{r}=0.70 \quad$ Loop gain at natural cycling

$\mathrm{p}=0.02$ frequency (fraction of placebo)

$\mathrm{r}=0.80$

$\mathrm{p}=0.003$
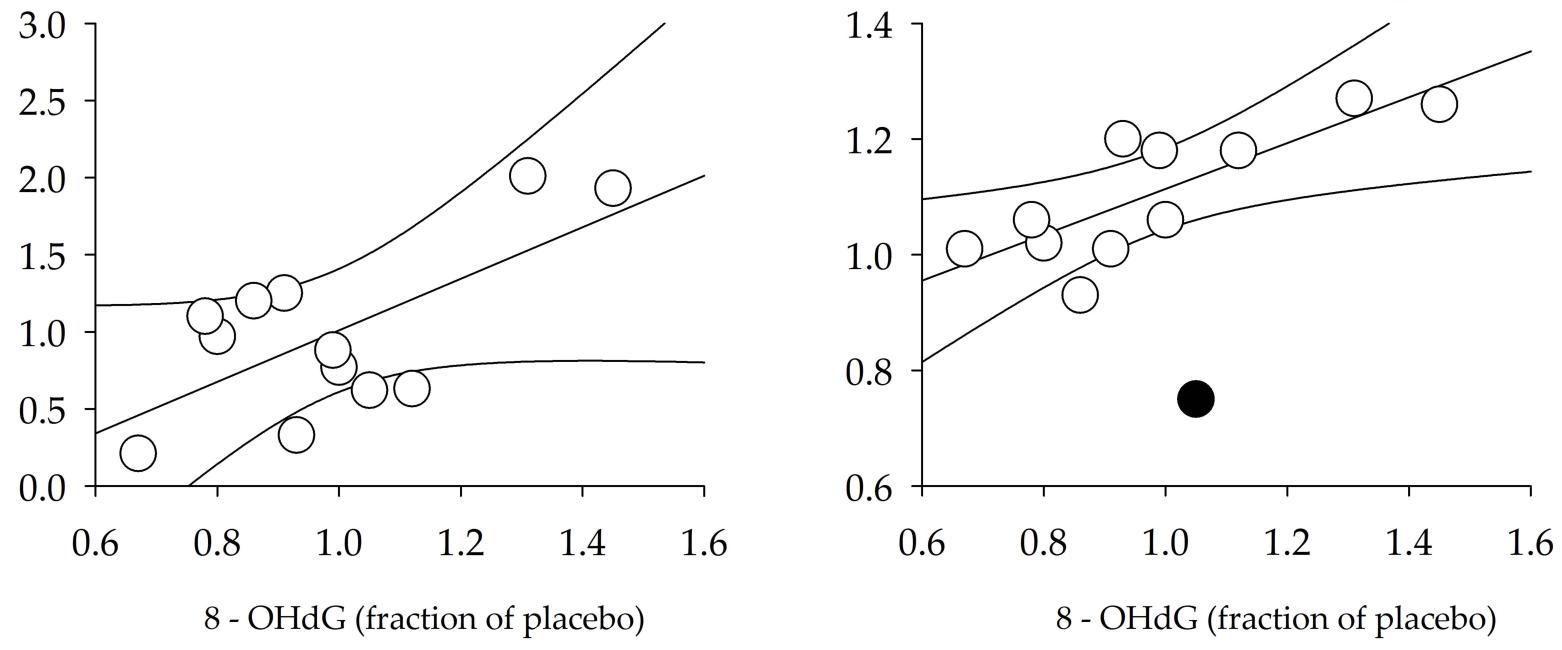

Figure 2 Scatterplots showing the correlation between measures of 8-hydroxyl-2-deoxy guanosine (8-OHdG) and measures of arousal, apnea severity (apnea/hypopnea index, number of events in a 7-min segment, percentage of segment in which breathing was stable and event duration) and loop gain. The data shown are measures obtained from the antioxidant trial expressed as a fraction of measures recorded for the placebo trial. $n=12$ participants. A Pearson correlation coefficient was used to complete the analysis. Black circle indicates a clear outlier. 

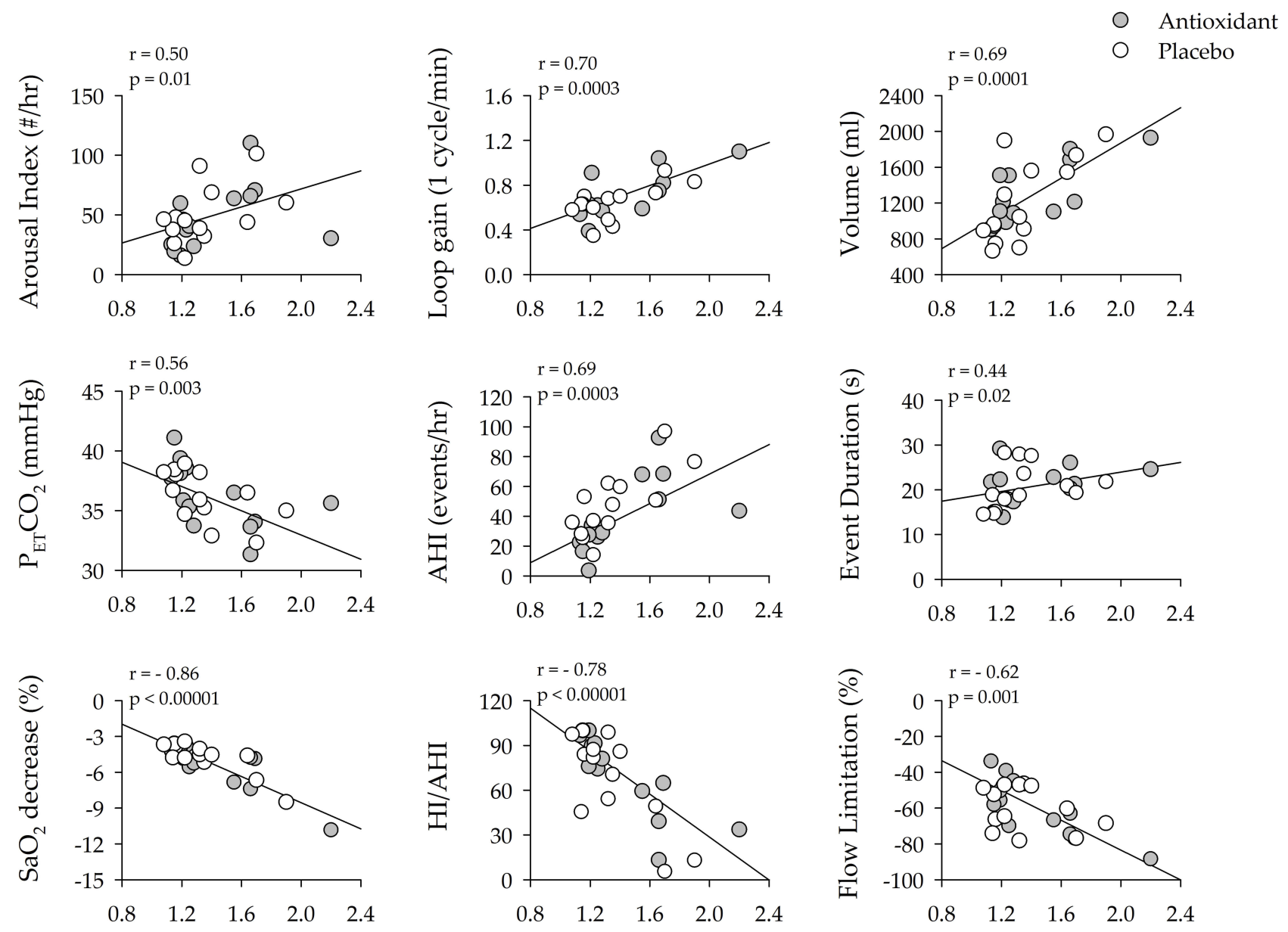

Arousal Threshold

Arousal Threshold

Arousal Threshold

Figure 3 Scatterplots showing the correlation between loop gain ( 1 cycle/s) and measures of arousal (arousal index, ventilatory response to arousal, end-tidal carbon dioxide following an arousal), apnea severity (apnea/hypopnea index, apnea duration and the severity of oxygen desaturation) and airway collapsibility (ratio of hypopneas/ apneas + hypopneas and the degree of flow limitation during hypopneas) during the antioxidant (gray circles) and placebo trials (white circles). Note that loop gain was significantly correlated to a number of indices of arousal and apnea severity. $n=13$ participants. A Pearson correlation coefficient was used to complete the analysis.

were negatively correlated to the decrease in oxygen saturation during breathing events (Figures 3 and 4). Lastly, the arousal threshold and $\mathrm{LG}_{1}$ were negatively correlated to the number of hypopneas expressed as a ratio of the apnea/hypopnea index and were negatively correlated to the degree of flow limitation (Figures 3 and 4).

Stepwise regression analyses were used to identify the variables that predicted indices of apnea severity and airway collapsibility. The arousal threshold, ventilatory response to arousal, the partial pressure of end-tidal carbon dioxide following arousal, $\mathrm{LG}_{1}, \mathrm{LG}_{\mathrm{n}}$, age and body mass index were used to predict indices of apnea severity and airway collapsibility including the apnea/hypopnea index, the decrease in oxygen saturation during an event, the hypopnea/apnea + hypopnea ratio and average flow limitation. In all cases, body mass index was forced into the equation. The results showed that the apnea/hypopnea index was predicted by a linear combination of the arousal threshold, $\mathrm{LG}_{1}$ and the partial pressure of endtidal carbon dioxide following an arousal (Table 2). Moreover, the decrease in oxygen saturation during an event was predicted by the arousal threshold and $\mathrm{LG}_{1}$ (Table 2). Alternatively, the average flow limitation obtained from each event and the hypopnea/apnea + hypopnea ratio was predicted by the arousal threshold and age (Table 2).

\section{Discussion}

Novel findings from our investigation include i) the positive correlation discovered between the change in oxidative stress during wakefulness and the change in loop gain during non-rapid eye movement sleep ii) the results which showed that a concomitant increase in both loop gain and the arousal threshold are clinical predictors of increased 


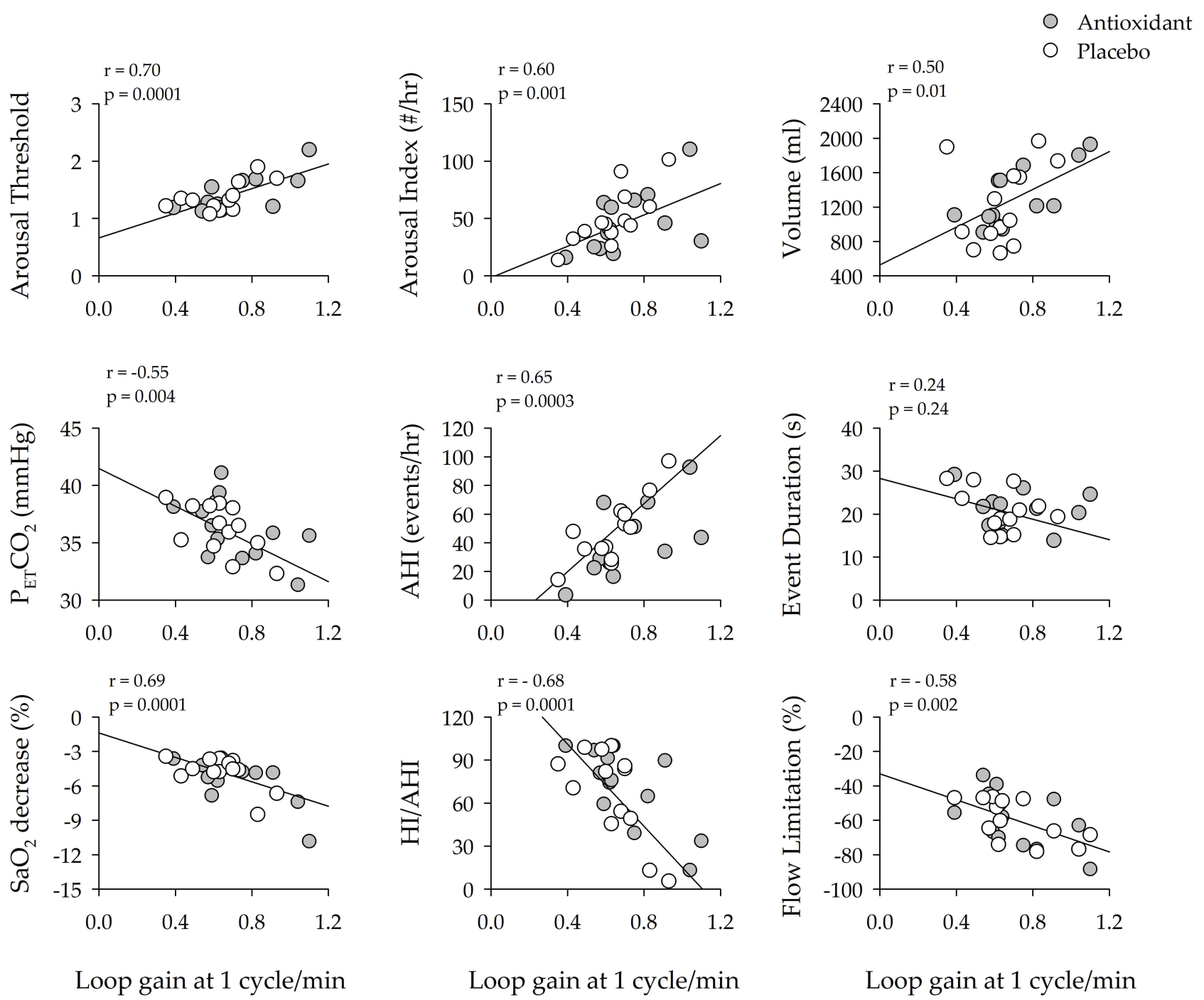

Figure 4 Scatterplots showing the correlation between arousal threshold and arousal severity (arousal index), the response to arousal (loop gain, ventilatory response to arousal, end-tidal carbon dioxide following an arousal), apnea severity (apnea/hypopnea index, apnea duration and the severity of oxygen desaturation) and airway collapsibility (the ratio of hypopneas/apneas + hypopneas, the degree of flow limitation during hypopneas) during the antioxidant (gray circles) and placebo trials (white circles). Note that arousal threshold was significantly correlated to a number of indices of arousal and apnea severity. $\mathrm{n}=\mathrm{I} 3$ participants. A Pearson correlation coefficient was used to complete the analysis.

apnea frequency and iii) the outcome which showed that an increase in the arousal threshold is a strong predictor of flow limitation.

\section{Oxidative Stress And Apnea Severity}

We hypothesized that accumulation of free radicals sensitizes the peripheral chemoreceptors resulting in an increase in controller gain and consequently loop gain during non-rapid eye movement sleep. Thus, we proposed that increases in oxidative stress during the placebo trial would result in an increase in loop gain, which would ultimately contribute to an increase in the ventilatory response at the termination of an arousal, compared to the antioxidant trial. We proposed that the increase in loop gain would be linked to increases in the apneahypopnea index and other measures of apnea severity, based on the mechanistic pathway outlined in the section "Introduction". However, our results showed that measures of loop gain, the arousal threshold, ventilatory response to arousal and the apnea/hypopnea index measured during sleep were similar during the antioxidant and placebo trial. If oxidative stress has a role in modifying mechanisms that impact apnea severity and airway collapsibility, the result is not surprising since average measures of oxidative stress were similar following exposure to intermittent hypoxia in the antioxidant and placebo trials. 


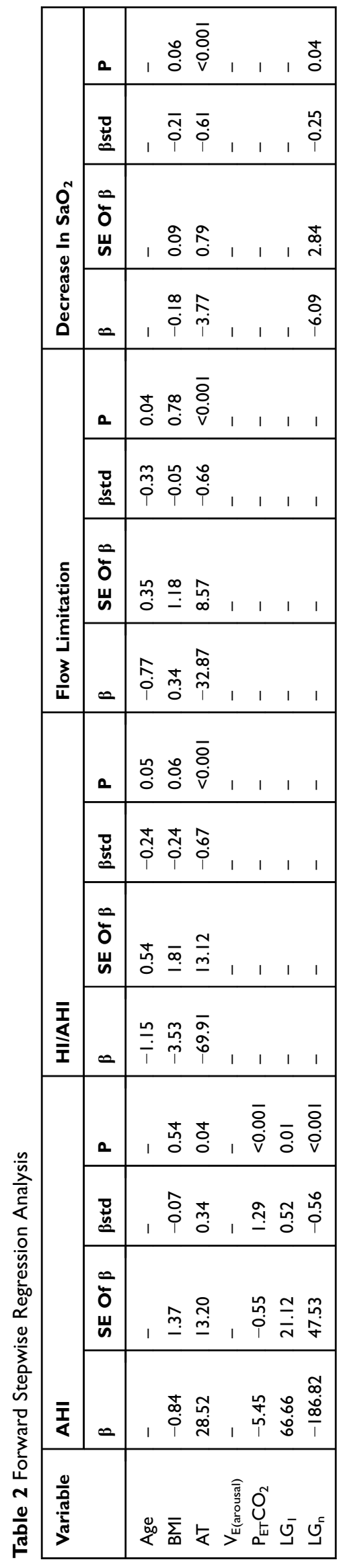

Nevertheless, once we expressed our measures from the antioxidant trial (see section "Oxidative Stress, Loop Gain, Arousal Threshold And Measures of Apnea Severity" for a detailed explanation) as a fraction of the measures from the placebo trial, it became clear that changes in oxidative stress were positively correlated to changes in loop gain in our participants during non-rapid eye movement sleep. To our knowledge, this finding is novel. As hypothesized, the increase in loop gain could be the result of increases in controller gain caused by increased peripheral chemoreceptor sensitivity. Our findings are supported by the findings of Pialoux et $\mathrm{al},{ }^{28}$ who showed that increases in oxidative stress in healthy awake males induced by exposure to intermittent hypoxia for 4 days was positively correlated to the hypoxic ventilatory response. Likewise, findings in rats have shown that the accumulation of free radicals enhances the carotid body sensory response to hypoxia ${ }^{11}$ and initiates carotid sensory nerve long-term facilitation. ${ }^{13}$ Increases in loop gain induced by oxidative stress may heighten apnea severity. ${ }^{29-31}$ Moreover, an increase in oxidative stress might dampen the output of medullary motor nerves that innervate upper airway muscles, ${ }^{32}$ which could impact apnea severity.

Independent of the mechanism, the link between oxidative stress and apnea severity is supported by our findings which showed that oxidative stress was correlated to a variety of measures of apnea severity (i.e., apnea-hypopnea index, number of events and arousals in 7-min segments, event duration, percentage of time breathing was stable). Our results add to previous findings which revealed that post-sleep measures of oxidative stress are correlated to the apnea-hypopnea index, nadir levels of oxygen saturation and the arousal index. ${ }^{33}$ The increase in obstructive sleep apnea severity, and the accompanying intermittent hypoxia, if severe, likely intensifies oxidative stress. As mentioned earlier, exposure to $6 \mathrm{hrs}$ of intermittent hypoxia daily for 4 consecutive days resulted in an increase in oxidative stress ${ }^{28}$ in humans. Likewise, indirect support for this hypothesis is found in studies which have shown that measures of oxidative stress are greater in individuals with obstructive sleep apnea compared to healthy individuals. ${ }^{17-21}$

\section{Loop Gain And Arousal Threshold As Clinical Markers Of Apnea Severity}

We previously proposed that increases in loop gain and/or a startle reflex ${ }^{15}$ might be responsible for initiating increases 
in ventilation at the termination of an arousal. This increase in ventilation, coupled with a concomitant reduction in carbon dioxide, could be responsible for perpetuating apneic events. ${ }^{29-31}$ This suggestion is supported by our univariate analysis which showed that the ventilatory response to arousal, and the accompanying reduction in the partial pressure of carbon dioxide, was correlated with a higher loop gain. Likewise, results from our stepwise regression analysis revealed that a greater loop gain and arousal threshold was correlated to the apnea-hypopnea index. This latter result was of interest since increases in loop gain coupled to reductions in the arousal threshold are typically postulated to be associated with increases in the apnea-hypopnea index (see below for further discussion). Our results also showed that increases in the arousal threshold were associated with the degree of flow limitation which we considered to be a surrogate marker of upper airway collapsibility.

This latter finding adds to the results of Edwards et al who showed, using measures of epiglottic pressure, that increases in the arousal threshold were negatively correlated to the percentage of hypopneas that contributed to the apnea/hypopnea index. ${ }^{34}$ Thus, there is support that increases in the arousal threshold may be associated with increased airway collapsibility, at least in some patients with obstructive sleep apnea. Indeed, additional preliminary data from our laboratory support this hypothesis. In a group of ten males with obstructive sleep apnea, we measured the arousal threshold during a baseline sleep study using the model employed in the present investigation. In a subsequent sleep study, we measured the critical closing pressure of the upper airway using a standard method that we have employed previously. ${ }^{35}$ Our results revealed a positive correlation between measures of the arousal threshold and the critical closing pressure (AT: $r=0.74$, $\mathrm{p}=0.02)$.

The question that has been presented in the past is whether or not modifications in the arousal threshold are a cause or a consequence of apneic events. ${ }^{34}$ Both a genetic predisposition and the subsequent consequences (e.g., intermittent hypoxia) of this disposition may be responsible for the relationship between the arousal threshold and the degree of flow limitation. We recently showed that exposure to mild intermittent hypoxia leads to increases in the arousal threshold. ${ }^{15}$ On the other hand, in many individuals with moderate sleep apnea, a low arousal threshold similar to that observed in healthy individuals is evident. ${ }^{34}$ Moreover, treatment with continuous positive airway pressure modifies the arousal threshold in individuals with obstructive sleep apnea but the response is variable. ${ }^{36}$ The likelihood of a pre-existing arousal threshold, that is subsequently modified by exposure to airway obstruction, leads to the intriguing possibility that the arousal threshold and upper airway collapsibility are mechanistically linked. For example, there are numerous modulators (e.g., serotonin) that impact both arousal from non-rapid eye movement sleep ${ }^{37}$ and the amplitude and frequency of upper airway motor neuron/ nerve discharge. ${ }^{38,39}$ Consequently, a given arousal threshold coupled to a given level of flow limitation may be a reflection of an inherent arousal state, similar to that experienced with the administration of anesthetic. ${ }^{40}$ Independent of the underlying explanation for the relationship, our results show that an increase in loop gain and/or an increase in the arousal threshold is linked to an increase in markers of apnea severity and airway collapsibility.

This relationship is divergent from published work which showed that a reduced arousal threshold contributes to increased apnea severity and airway collapsibility in at least $50 \%$ of the individuals with obstructive sleep. ${ }^{34,41,42}$ At least three potential mechanisms have been suggested to be responsible for the link between a reduced arousal threshold and increased apnea severity. ${ }^{34}$ The relationship could exist because a sufficient amount of time required for respiratory stimuli to recruit pharyngeal muscles and re-open the airway before arousal is not obtainable, because of the low arousal threshold. ${ }^{41}$ Given this premise, a low arousal threshold could be coupled to a more collapsible airway. If so, the arousal index (respiratory and non-respiratory index) should be higher and indices of airway collapsibility (i.e., the degree of airflow limitation, the amount of time associated with stable breathing and the percentage of apnea or hypopnea events as a percentage of total events) would signify a propensity towards more unstable breathing.

In addition to the potential impact that the arousal threshold has on the recruitment of pharyngeal muscles, repeated arousal caused by a low arousal threshold may be coupled to a robust ventilatory response, ${ }^{43}$ in some cases. If this occurs, then a low arousal threshold would be expected to be accompanied by an increased arousal index, coupled to elevations in the ventilatory response at the termination of arousal and greater reductions in the partial pressure of end-tidal carbon dioxide.

Lastly, a low arousal threshold could also lead to increased sleep fragmentation, ${ }^{41}$ which would prevent individuals from achieving sleep associated with a stable 
airway. Given this scenario, an increase in the percentage of non-stable sleep (i.e., N1 and N2) and a reduction in total sleep time would be expected to be coupled to a low arousal threshold.

Based on the proposed outcomes associated with a low arousal threshold (e.g., increased airway collapsibility, increased ventilatory response to arousal and hypocapnia, decreased total sleep time) one would anticipate improvement in apnea severity with an increase in the arousal threshold. However, our findings indicate that a lower arousal index is not always coupled to increases in apnea severity. Our results and previous findings ${ }^{34}$ suggest that decreases in the arousal threshold serve to prevent apnea (i.e., healthy individuals) or limit the severity of apnea at least in a subpopulation of individuals with sleep apnea.

\section{Translational Significance}

Our results have shown that increases in oxidative stress are associated with increased loop gain and exacerbated measures of obstructive sleep apnea severity. Whether or not the impact of oxidative stress on apnea severity is mediated solely by increases in loop gain or by other mechanisms, including a direct effect on the functioning of upper airway motoneurons (e.g., hypoglossal motoneurons) requires further exploration. Likewise, additional studies are required to address the efficacy of antioxidants in mitigating apnea severity.

An increase in loop gain and/or the arousal threshold were correlated to measures of apnea severity and airway collapsibility. The positive correlation between the arousal threshold and degree of upper airway collapsibility is intriguing and provides the impetus for further exploration. This exploration could include investigating if individuals with a higher arousal threshold require greater continuous positive airway pressure to eliminate apneic events. Likewise, future studies might explore if those with a higher arousal threshold are more tolerant and compliant with continuous positive airway. In the absence of continuous positive airway pressure our results suggest that in some individuals with sleep apnea, a reduction in the arousal threshold may be beneficial in reducing the severity of sleep apnea.

\section{Limitations}

Our participants were recruited on a first-come basis. As a result, the population recruited was composed of males and the associations reported may or may not be similar in females. The participants did not suffer from other co-morbid conditions. Thus, from a favorable point of view, the potential influences of other co-morbidities on the reported associations were eliminated. On the other hand, the current findings might not be generalizable to the wider population with obstructive sleep apnea.

Measures of oxidative stress following exposure to mild intermittent hypoxia were similar during the antioxidant and placebo trials. This similarity occurred despite reductions in oxidative stress following exposure to intermittent hypoxia during the antioxidant trial because baseline levels of oxidative stress were elevated in some participants. The reason that baseline measures were increased in some participants is not clear. However, participants were untreated, and diet and exercise between trials were uncontrolled; consequently, these variables may have impacted on baseline values.

In a few participants, measures of oxidative stress increased during recovery compared to baseline. An increased dose of the antioxidant cocktail may have been required to improve efficacy in those participants that experienced an increase in oxidative stress.

Despite exposure to mild intermittent hypoxia, measures of oxidative stress did not increase dramatically in the group as a whole during recovery compared to baseline in the placebo trial. The mild intermittent hypoxia protocol employed in the present investigation was used because it was shown to initiate various forms of respiratory plasticity in prior studies ${ }^{1,6}$ and was associated with an increase in oxidative stress in a prior investigation. ${ }^{6}$ Further investigation is required to determine the optimal number of episodes and hypoxic intensity to initiate a desired level of oxidative stress.

\section{Ethics Approval}

Participants were informed about the requirements, benefits and potential risks of the study before providing their written informed consent to participate. The experimental procedures adhered to the standards set by the Declaration of Helsinki and were approved by the Human Investigations Committees of Wayne State University and John D. Dingell Veterans Affairs Medical Centre (No. 070103M1F).

\section{Acknowledgments}

We thank Scott A. Sands PhD for making available the software program that was used to quantify loop gain and the arousal threshold. We also thank Dr. Sands for his comments on the manuscript. 


\section{Author Contributions}

All authors contributed to data analysis, drafting or revising the article, gave final approval of the version to be published, and agree to be accountable for all aspects of the work.

\section{Funding}

This work was supported by awards (I01CX000125 and 15SRCS003) from the Department of Veterans Affairs, Veterans Health Administration, Office of Research and Development, and awards (R01HL085537, R56HL142757) from the National Heart, Lung and Blood Institutes.

\section{Disclosure}

The authors report no conflicts of interest in this work.

\section{References}

1. Gerst DG III, Yokhana SS, Carney LM, et al. The hypoxic ventilatory response and ventilatory long-term facilitation are altered by time of day and repeated daily exposure to intermittent hypoxia. $J$ Appl Physiol. 2011;110(1):15-28. doi:10.1152/japplphysiol.00524.2010

2. Khodadadeh B, Badr MS, Mateika JH. The ventilatory response to carbon dioxide and sustained hypoxia is enhanced after episodic hypoxia in OSA patients. Respir Physiol Neurobiol. 2006;150(23):122-134. doi:10.1016/j.resp.2005.04.019

3. Mateika JH, Mendello C, Obeid D, Badr MS. Peripheral chemoreflex responsiveness is increased at elevated levels of carbon dioxide after episodic hypoxia in awake humans. J Appl Physiol. 2004;96 (3):1197-1205. doi:10.1152/japplphysiol.00573.2003

4. Morelli C, Badr MS, Mateika JH. Ventilatory responses to carbon dioxide at low and high levels of oxygen are elevated after episodic hypoxia in men compared with women. J Appl Physiol. 2004;97 (5):1673-1680. doi:10.1152/japplphysiol.00541.2004

5. Harris DP, Balasubramaniam A, Badr MS, Mateika JH. Long-term facilitation of ventilation and genioglossus muscle activity is evident in the presence of elevated levels of carbon dioxide in awake humans. Am J Physiol Regul Integr Comp Physiol. 2006;291(4):R1111R1119. doi:10.1152/ajpregu.00896.2005

6. Lee DS, Badr MS, Mateika JH. Progressive augmentation and ventilatory long-term facilitation are enhanced in sleep apnoea patients and are mitigated by antioxidant administration. $J$ Physiol. 2009;587 (Pt 22):5451-5467. doi:10.1113/jphysiol.2009.178053

7. Syed Z, Lin HS, Mateika JH. The impact of arousal state, sex, and sleep apnea on the magnitude of progressive augmentation and ventilatory long-term facilitation. $J$ Appl Physiol. 2013;114(1):52-65. doi:10.1152/japplphysiol.00985.2012

8. Wadhwa H, Gradinaru C, Gates GJ, Badr MS, Mateika JH. Impact of intermittent hypoxia on long-term facilitation of minute ventilation and heart rate variability in men and women: do sex differences exist? J Appl Physiol. 2008;104(6):1625-1633. doi:10.1152/ japplphysiol.01273.2007

9. Chowdhuri S, Pierchala L, Aboubakr SE, Shkoukani M, Badr MS. Long-term facilitation of genioglossus activity is present in normal humans during NREM sleep. Respir Physiol Neurobiol. 2008;160 (1):65-75. doi:10.1016/j.resp.2007.08.007

10. El-Chami M, Sudan S, HS L, Mateika JH. Exposure to intermittent hypoxia and sustained hypercapnia reduces therapeutic CPAP in participants with obstructive sleep apnea. J Appl Physiol. 2017;123 (4):993-1002. doi:10.1152/japplphysiol.00204.2017
11. Peng YJ, Prabhakar NR. Effect of two paradigms of chronic intermittent hypoxia on carotid body sensory activity. J Appl Physiol. 2004;96(3):1236-1242. doi:10.1152/japplphysiol.00820.2003

12. Peng YJ, Prabhakar NR. Reactive oxygen species in the plasticity of respiratory behavior elicited by chronic intermittent hypoxia. $J$ Appl Physiol. 2003;94(6):2342-2349. doi:10.1152/japplphysiol.00613.2002

13. Peng YJ, Overholt JL, Kline D, Kumar GK, Prabhakar NR. Induction of sensory long-term facilitation in the carotid body by intermittent hypoxia: implications for recurrent apneas. Proc Natl Acad Sci U S A. 2003;100(17):10073-10078. doi:10.1073/pnas.1734109100

14. Wilkerson JE, Satriotomo I, Baker-Herman TL, Watters JJ, Mitchell GS. Okadaic acid-sensitive protein phosphatases constrain phrenic long-term facilitation after sustained hypoxia. $J$ Neurosci. 2008;28 (11):2949-2958. doi:10.1523/JNEUROSCI.5539-07.2008

15. Alex RM, Panza GS, Hakim H, et al. Exposure to mild intermittent hypoxia increases loop gain and the arousal threshold in participants with obstructive sleep apnoea. J Physiol. 2019;597(14):3697-3711. doi:10.1113/JP277711

16. Wains SA, El-Chami M, Lin HS, Mateika JH. Impact of arousal threshold and respiratory effort on the duration of breathing events across sleep stage and time of night. Respir Physiol Neurobiol. 2017;237:35-41. doi:10.1016/j.resp.2016.12.009

17. Carpagnano GE, Kharitonov SA, Resta O, Foschino-Barbaro MP, Gramiccioni E, Barnes PJ. 8-Isoprostane, a marker of oxidative stress, is increased in exhaled breath condensate of patients with obstructive sleep apnea after night and is reduced by continuous positive airway pressure therapy. Chest. 2003;124(4):1386-1392. doi:10.1378/chest.124.4.1386

18. Jurado-Gamez B, Fernandez-Marin MC, Gomez-Chaparro JL, et al. Relationship of oxidative stress and endothelial dysfunction in sleep apnoea. Eur Respir J. 2011;37(4):873-879. doi:10.1183/09031936. 00027910

19. Lavie L, Vishnevsky A, Lavie P. Evidence for lipid peroxidation in obstructive sleep apnea. Sleep. 2004;27(1):123-128.

20. Mancuso M, Bonanni E, LoGerfo A, et al. Oxidative stress biomarkers in patients with untreated obstructive sleep apnea syndrome. Sleep Med. 2012;13(6):632-636. doi:10.1016/j.sleep.2011.10.030

21. Shi T, Min M, Sun C, et al. A meta-analysis of the association between gout, serum uric acid level, and obstructive sleep apnea. Sleep Breath. 2019. doi:10.1007/s11325-019-01827-1

22. Berry RB, Budhiraja R, Gottlieb DJ, et al. Rules for scoring respiratory events in sleep: update of the 2007 AASM manual for the scoring of sleep and associated events. deliberations of the sleep apnea definitions task force of the American Academy of Sleep Medicine. J Clin Sleep Med. 2012;8(5):597-619. doi:10.5664/jcsm.2172

23. Terrill PI, Edwards BA, Nemati S, et al. Quantifying the ventilatory control contribution to sleep apnoea using polysomnography. Eur Respir J. 2015;45(2):408-418. doi:10.1183/09031936.00062914

24. Joosten SA, Leong P, Landry SA, et al. Loop gain predicts the response to upper airway surgery in patients with obstructive sleep apnea. Sleep. 2017;40:7. doi:10.1093/sleep/zsx094

25. Landry SA, Andara C, Terrill PI, et al. Ventilatory control sensitivity in patients with obstructive sleep apnea is sleep stage dependent. Sleep. 2018;41:5. doi:10.1093/sleep/zsy024

26. Berry RB, Asyali MA, McNellis MI, Khoo MC. Within-night variation in respiratory effort preceding apnea termination and EEG delta power in sleep apnea. J Appl Physiol. 1998;85(4):1434-1441. doi:10.1152/jappl.1998.85.4.1434

27. Ratnavadivel R, Stadler D, Windler S, et al. Upper airway function and arousability to ventilatory challenge in slow wave versus stage 2 sleep in obstructive sleep apnoea. Thorax. 2010;65(2):107-112. doi:10.1136/thx.2008.112953

28. Pialoux V, Hanly PJ, Foster GE, et al. Effects of exposure to intermittent hypoxia on oxidative stress and acute hypoxic ventilatory response in humans. Am J Respir Crit Care Med. 2009;180 (10):1002-1009. doi:10.1164/rccm.200905-0671OC 
29. Mateika JH, Narwani G. Intermittent hypoxia and respiratory plasticity in humans and other animals: does exposure to intermittent hypoxia promote or mitigate sleep apnoea? Exp Physiol. 2009;94 (3):279-296. doi:10.1113/expphysiol.2008.045153

30. Mateika JH, Sandhu KS. Experimental protocols and preparations to study respiratory long term facilitation. Respir Physiol Neurobiol. 2011;176(1-2):1-11. doi:10.1016/j.resp.2011.01.007

31. Mateika JH, Syed Z. Intermittent hypoxia, respiratory plasticity and sleep apnea in humans: present knowledge and future investigations Respir Physiol Neurobiol. 2013;188(3):289-300. doi:10.1016/j. resp.2013.04.010

32. Veasey SC, Zhan G, Fenik P, Pratico D. Long-term intermittent hypoxia: reduced excitatory hypoglossal nerve output. Am J Respir Crit Care Med. 2004;170(6):665-672. doi:10.1164/rccm.200403-261OC

33. Chua AP, Aboussouan LS, Minai OA, Paschke K, Laskowski D, Dweik RA. Long-term continuous positive airway pressure therapy normalizes high exhaled nitric oxide levels in obstructive sleep apnea. J Clin Sleep Med. 2013;9(6):529-535. doi:10.5664/jcsm.2740

34. Edwards BA, Eckert DJ, McSharry DG, et al. Clinical predictors of the respiratory arousal threshold in patients with obstructive sleep apnea. Am J Respir Crit Care Med. 2014;190(11):1293-1300. doi:10.1164/rccm.201404-0718OC

35. El-Chami M, Shaheen D, Ivers B, et al. Time of day affects the frequency and duration of breathing events and the critical closing pressure during NREM sleep in participants with sleep apnea. $J$ Appl Physiol. 2015;119(6):617-626. doi:10.1152/japplphysiol.00346.2015
36. Loewen A, Ostrowski M, Laprairie J, et al. Determinants of ventilatory instability in obstructive sleep apnea: inherent or acquired? Sleep. 2009;32(10):1355-1365. doi:10.1093/sleep/32.10.1355

37. Solarewicz JZ, Angoa-Perez M, Kuhn DM, Mateika JH. The sleepwake cycle and motor activity, but not temperature, are disrupted over the light-dark cycle in mice genetically depleted of serotonin. Am J Physiol Regul Integr Comp Physiol. 2015;308(1):R10-R17. doi:10.1152/ajpregu.00400.2014

38. Fenik P, Veasey SC. Pharmacological characterization of serotonergic receptor activity in the hypoglossal nucleus. Am J Respir Crit Care Med. 2003;167(4):563-569. doi:10.1164/rccm.200202-107OC

39. Kubin L, Mann GL. Hypoglossal motoneurons are endogenously activated by serotonin during the active period of circadian cycle. Respir Physiol Neurobiol. 2018;248:17-24. doi:10.1016/j.resp.2017.11.002

40. Hillman DR, Platt PR, Eastwood PR. Anesthesia, sleep, and upper airway collapsibility. Anesthesiol Clin. 2010;28(3):443-455. doi:10.1016/j.anclin.2010.07.003

41. Eckert DJ, Younes MK. Arousal from sleep: implications for obstructive sleep apnea pathogenesis and treatment. $J$ Appl Physiol. 2014;116(3):302-313. doi:10.1152/japplphysiol.00649.2013

42. Osman AM, Carter SG, Carberry JC, Eckert DJ. Obstructive sleep apnea: current perspectives. Nat Sci Sleep. 2018;10:21-34. doi:10.2147/NSS.S124657

43. Younes M. Role of respiratory control mechanisms in the pathogenesis of obstructive sleep disorders. Journal Appl Physiol. 2008;105 (5):1389-1405. doi:10.1152/japplphysiol.90408.2008

\section{Publish your work in this journal}

Nature and Science of Sleep is an international, peer-reviewed, open access journal covering all aspects of sleep science and sleep medicine, including the neurophysiology and functions of sleep, the genetics of sleep, sleep and society, biological rhythms, dreaming, sleep disorders and therapy, and strategies to optimize healthy sleep.
The manuscript management system is completely online and includes a very quick and fair peer-review system, which is all easy to use. Visit http://www.dovepress.com/testimonials.php to read real quotes from published authors. 